\title{
Caracterización de bacterias cultivables aisladas de sitios de arrecifes mesofóticos de Cuba
}

\section{Characterization of culturable bacteria isolated from mesophotic reef sites in Cuba}

\author{
Eudalys Ortiz Guilarte ${ }^{*}$, María Victoria Iglesias Rodríguez ${ }^{1}$, Roberto Rafael Núñez Moreira ${ }^{1}$, \\ Valia Caballero Barreto ${ }^{1}$, Daymarlen González Tamayo ${ }^{1}$ \& \\ Gladys Margarita Lugioyo Gallardo ${ }^{1^{*}}$
}

\begin{abstract}
RESUMEN
Las comunidades bacterianas de sitios de arrecifes mesofóticos constituyen información genética de interés por las condiciones particulares de temperatura, presión, salinidad y disponibilidad de materia orgánica y nutrientes en la que se desarrollan. El presente trabajo tuvo como objetivo evaluar la diversidad bacteriana en aguas aledañas a los arrecifes mesofóticos de Cuba en mayo-junio de 2017; así como algunas de sus potencialidades biotecnológicas. Los cultivos fueron aislados en medio agar marino, en dos niveles de la columna de agua, en 10 sitios de muestreos. Posteriormente se evaluaron las capacidades metabólicas de los aislados, relacionadas con la asimilación de diferentes fuentes de carbono y la producción de metabolitos bioactivos. Los resultados permitieron la caracterización de 30 aislados bacterianos de aguas del nivel subsuperficial y 32 de aguas profundas. El mayor porcentaje corresponde a bacilos esporulados Gram positivos y bacilos Gram negativos. Asimismo, se demostró que el 85.5\% degradan carbohidratos de diferente naturaleza y el $96.8 \%$ producen enzimas hidrolíticas con actividad caseinasa, gelatinasa, lipasa o amilasa. La capacidad de degradar petróleo y compuestos fenólicos indistintamente fue detectada en el $61.3 \%$ de los aislados. Entre las potencialidades de interés biomédico e industrial se detectó actividad tensioactiva y asparaginasa en el 53\% y $32 \%$ de los cultivos, respectivamente. Por su parte, la caracterización taxonómica de 40 cultivos seleccionados por sus potencialidades metabólicas demostró que el género Bacillus presentó mayor actividad biológica. Estos resultados constituyen el primer acercamiento a la caracterización de la diversidad bacteriana en sitios de arrecifes mesofóticos de Cuba.
\end{abstract}

Palabras claves: aguas profundas, bacterioplancton, degradación, enzimas hidrolíticas, metabolitos

1 Instituto de Ciencias del Mar (ICIMAR), calle Loma \# 14, entre 35 y 37, Plaza de la Revolución, La Habana, Cuba. laly@icimar.cu* ORCID: https://orcid.org/0000-0001-8043-4076, victoria@icimar.cu ORCID: https://orcid. org/0000-0002-1548-4285, robertico@icimar.cu ORCID: https://orcid.org/0000-0001-7327-9442, valia@icimar. cu ORCID: https://orcid.org/0000-0002-6452-2065, mimi@icimar.cu ORCID: https://orcid.org/0000-0002-46531347,margarita@icimar.cu* ORCID: https://orcid.org/0000-0001-7517-8063 


\section{ABSTRACT}

The bacterial communities of mesophotic reef sites constitute genetic information of interest due to the particular conditions of temperature, pressure, salinity and availability of organic matter and nutrients in the environment in which they develop. The present work aimed to evaluate the bacterial diversity in waters surrounding the mesophotic reefs of Cuba during the period May-June 2017; as well as some of its biotechnological potentialities. Bacterial cultures were isolated on marine agar medium for two water column depth levels at 10 sampling sites. Subsequently, the metabolic capacities of the isolates were evaluated and related to the assimilation of different carbon sources and the production of bioactive metabolites. The results allowed the characterization of 30 bacterial cultures from subsurface-level and 32 from deep-sea waters. The highest percentage corresponds to Gram positive $\left(\mathrm{G}^{+}\right)$sporulated bacilli and Gram negative (G-) bacilli. Likewise, it was shown that $85.5 \%$ of the isolates degrade carbohydrates of different nature and $96.8 \%$ produce hydrolytic enzymes with caseinase, gelatinase, lipase or amylase activity. The ability to degrade petroleum oil and phenolic compounds indistinctly was detected in $61.3 \%$ of the isolates. Among the potentialities of biomedical and industrial interest, surfactant and L-asparaginase activity was detected in $53 \%$ and $32 \%$ of the cultures, respectively. For its part, the taxonomic characterization of 40 cultures selected for their metabolic potentials showed that the genus Bacillus presented greater biological activity. These results constitute the first approach to the characterization of bacterial diversity in mesophotic reef sites of Cuba.

Keywords: Deep-sea waters, bacterioplankton, degradation, hydrolytic enzymes, metabolites

\section{INTRODUCCIÓN}

\section{Las comunidades microbianas} en los ecosistemas marinos desempeñan un papel importante en la mineralización de materia orgánica, así como en la transferencia de energía a otros niveles tróficos (Sivaperumal et al. 2017; Liu et al. 2018; Cui et al. 2019). Además, los microorganismos son un componente esencial dentro de la diversidad biológica por su elevada diversidad genética y metabólica para la búsqueda de nuevos compuestos con aplicaciones biotecnológicas (Glöckner et al. 2012; Prabhu et al. 2017).

En la literatura especializada está documentado que la diversidad genética y la actividad de las bacterias marinas planctónicas están significativamente influenciadas por las condiciones ambientales (Cui et al. 2019). Diversas investigaciones han demostrado que la composición de la comunidad bacteriana en los ecosistemas depende de las condiciones físico-químicas tales como temperatura, salinidad, disponibilidad de materia orgánica y nutrientes (Gilbert et al. 2012; Ward et al. 2017).

En Cuba desde el punto de vista microbiológico solo se han realizado investigaciones de calidad de las aguas en los arrecifes someros del suroeste (SW) de la Isla (Miravet, 2003), en el archipiélago Sabana-Camagüey, ubicado en la zona norcentral de Cuba (Miravet et al. 1994) y más recientemente en los arrecifes del golfo de Cazones, SW de Cuba (Lugioyo et al. 2020), por lo que este estudio constituye el primer acercamiento al conocimiento de la 
microbiota aledaña a sitios de arrecifes mesofóticos de Cuba.

Los ecosistemas coralinos mesofóticos son comunidades arrecifales caracterizadas por la presencia de corales fotodependientes y comunidades asociadas en regiones tropicales y subtropicales. Estos arrecifes presentan una menor diversidad de especies en comparación con los arrecifes someros, pero comúnmente tienen mayores porcentajes de cobertura coralina (4060\%) (Olson \& Kellogs, 2010). La diversidad de los arrecifes mesofóticos resulta poco conocida en la región del Caribe (Loya et al. 2016; Roman, 2018) y en Cuba, nunca antes se habían estudiado debido a las dificultades que impone la colecta y toma de muestras a estas profundidades.

El objetivo del presente trabajo fue evaluar algunas de capacidades metabólicas de aislados bacterianos de aguas aledañas a arrecifes mesofóticos de Cuba; así como la caracterización taxonómica de los cultivos más promisorios.

\section{MATERIALES Y MÉTODOS}

La expedición científica conjunta Cuba-Estados Unidos fue realizada entre el 15 de mayo y 11 de junio de 2017, donde se muestrearon 10 sitios de arrecifes mesofóticos de Cuba (Cuadro 1). Las muestras para los análisis microbiológicos se colectaron en frascos de vidrio estériles de $250 \mathrm{~mL}$ de capacidad y se tomaron de la capa subsuperficial $(5 \mathrm{~m})$ y de las aguas de las máximas profundidades (135-450 m); utilizando una roseta con botellas oceanográficas Niskin de 10 L de capacidad.

Cuadro 1. Georreferenciación de los sitios de muestreo de los arrecifes mesofóticos alrededor de Cuba

Table 1. Georeferencing of mesophotic reef sampling sites around Cuba

\begin{tabular}{lllll}
\hline Estaciones & Región & $\begin{array}{l}\text { Profundidad } \\
(\mathbf{m})\end{array}$ & Latitud & Longitud \\
\hline C-04 & NW & 397 & $22^{\circ} 53.9640^{\prime}$ & $83^{\circ} 35.1060^{\prime}$ \\
C-10 & NW & 358 & $22^{\circ} 02.3900^{\prime}$ & $85^{\circ} 04.0550^{\prime}$ \\
$\mathbf{C - 2 3}$ & SW & 450 & $21^{\circ} 29.2540^{\prime}$ & $82^{\circ} 39.3100^{\prime}$ \\
$\mathbf{C - 2 8}$ & SW & 315 & $21^{\circ} 33.4260^{\prime}$ & $81^{\circ} 44.4540^{\prime}$ \\
$\mathbf{C - 3 3}$ & SW & 350 & $22^{\circ} 07.6440^{\prime}$ & $81^{\circ} 07.7400^{\prime}$ \\
C-48 & SE & 320 & $19^{\circ} 49.4000^{\prime}$ & $77^{\circ} 44.0000^{\prime}$ \\
C-50 & SE & 301 & $19^{\circ} 56.4000^{\prime}$ & $76^{\circ} 24.0000^{\prime}$ \\
C-53 & NE & 280 & $20^{\circ} 19.1000^{\prime}$ & $74^{\circ} 16.2999^{\prime}$ \\
C-54 & NE & 253 & $21^{\circ} 05.5400^{\prime}$ & $75^{\circ} 39.6500^{\prime}$ \\
C-58 & NE & 135 & $22^{\circ} 34.0000^{\prime}$ & $78^{\circ} 23.6000^{\prime}$ \\
\hline
\end{tabular}




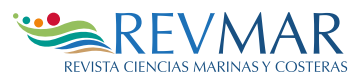

Para el aislamiento de los cultivos puros de bacterias heterótrofas se utilizó el medio ZoBell 2216E (Oppenheimer \& ZoBell, 1952) de acuerdo a la metodología propuesta por Miravet et al. (2009) y el criterio de selección de las colonias fue la frecuencia de aparición de características culturales diferentes. Los cultivos axénicos fueron conservados según la metodología establecida por Benson (2001) y depositados en la Colección de Bacterias Marinas del ICIMAR.

Para la caracterización metabólica de los aislados fue evaluada la asimilación de carbohidratos como fuentes de carbono, suplementado con glucosa, manosa, sacarosa, lactosa y almidón al $1.0 \%$, de acuerdo a las metodologías descritas por Atlas (2010). Además, se evaluó el crecimiento microbiano en compuestos xenobióticos en medio Vela \& Ralston (1978) agarizado y utilizando indistintamente como fuente de carbono petróleo Mesa $30(10 \mathrm{~g} / \mathrm{mL})$, fenol y pentaclorofenol $(1 \mathrm{~g} / \mathrm{mL})$. Las condiciones de cultivos fueron establecidas según la metodología de Miravet et al. (2009).

La evaluación de la producción de enzimas hidrolíticas se realizó a partir del crecimiento microbiano (Harrigan \& McCance, 1968). Para la actividad caseinasa, amilasa y lipasa los cultivos fueron inoculados en placas Petri con medios suplementados indistintamente con caseína, almidón y Tween 80 al $1 \%$. La actividad geletinasa se evaluó mediante la siembra por punción del medio con gelatina al $1 \%$. Todos los ensayos fueron incubados a $30 \pm 2^{\circ} \mathrm{C}$ durante 24 o $72 \mathrm{~h}$ de acuerdo a la experiencia. La actividad se evaluó por la formación de halos alrededor de la colonia, excepto para la gelatina determinada por la licuación del medio luego del crecimiento microbiano. La determinación semicuantitativa de la actividad caseinasa, amilasa y lipasa se calculó por la diferencia entre el diámetro del halo y el diámetro de la colonia (Dias et al. 2009).

La actividad asparaginasa fue detectada mediante el crecimiento en placas Petri con medio Glicerol Asparagina Agar suplementado con $0.3 \mathrm{~mL}$ de una solución de rojo fenol al $2.5 \%$ y el $\mathrm{pH}$ ajustado a 6.5. Los cultivos fueron incubados a $30 \pm 2^{\circ} \mathrm{C}$ durante cinco días, considerando como positivo la aparición de un halo de coloración rosado alrededor del crecimiento microbiano (Sivasankar et al. 2013).

La producción de tensioactivos se determinó a partir del cultivo fermentado en el medio propuesto por Finnerty (1994) y suplementado con sacarosa al $2 \%$; durante $18 \mathrm{~h}$ a $125 \mathrm{r} / \min$ y $30 \pm 2^{\circ} \mathrm{C}$ en zaranda orbital (Infors HT Ecotron). Las determinaciones se realizaron a los cultivos libres de células, obtenidos por centrifugación a 7000 xg y $4{ }^{\circ} \mathrm{C}$ durante 30 min., en una centrífuga Beckman J2-H5; mediante el método de anillo de Du Noüy en un tensiómetro Krüss K 10 T. Como control negativo se empleó el 
medio de cultivo. Los cultivos con valores de tensión superficial $\leq 35 \mathrm{mN} / \mathrm{m}$ fueron considerados como productores de tensioactivos.

La caracterización taxonómica de los cultivos seleccionados por sus capacidades metabólicas fue realizada mediante el sistema API con la utilización del software de identificación bacteriana APILAB Plus. Para el grupo de los bacilos Gram negativos (G-) no enterobacterias se utilizaron las tiras API 20NE, mientras para el estudio metabólico de carbohidratos y la identificación de bacilos Gram positivos $(\mathrm{G}+)$ esporulados se utilizó el medio API 50CHB/E, API 50CH y API $20 \mathrm{E}$.

El análisis de similitud de los aislados seleccionados se realizó por métodos aglomerativos jerárquicos mediante el coeficiente de similitud de Brays-Curtis empleando el programa estadístico PRIMER 6.1.6. (Clarke \& Warwick, 2001).

\section{RESULTADOS}

A partir de las características culturales se obtuvieron 62 aislados de bacterias heterótrofas, 30 pertenecen a las aguas del nivel subsuperficial y 32 a las aguas profundas de los sitios de arrecifes mesofóticos de Cuba. La caracterización microscópica de los cultivos evidenció que el 50\% de los aislados del nivel subsuperficial pertenecían al grupo de los bacilos $\mathrm{G}+$ esporulados y en menor proporción los bacilos Gram positivos asporógenos $(27.0 \%)$ y los bacilos G$(23.0 \%)$. En las aguas del nivel de fondo predominaron los bacilos G- (53.0\%), seguido de los bacilos esporulados $\mathrm{G}+$ $(44.0 \%)$ y en un porcentaje bajo los bacilos $\mathrm{G}+$ asporógenos (Fig. 1).
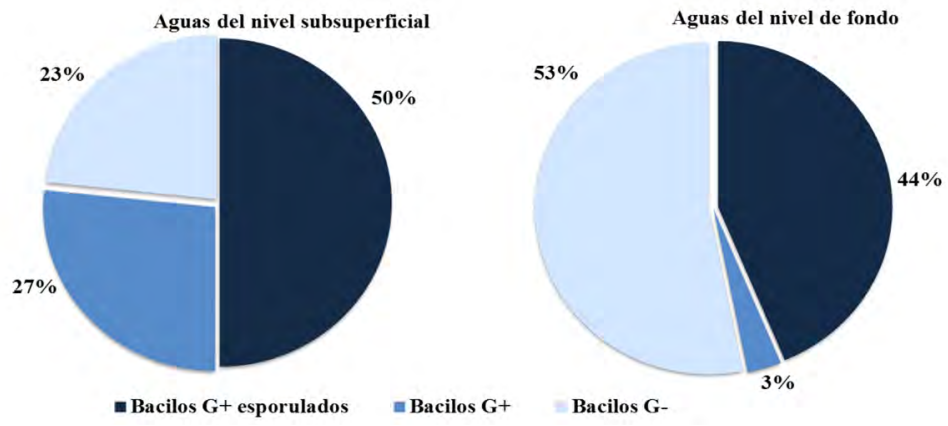

Fig. 1. Distribución de los grupos fenotípicos de las bacterias heterótrofas aisladas del nivel subsuperficial $(5 \mathrm{~m}) \mathrm{y}$ de las aguas profundas de sitios de arrecifes mesofóticos de Cuba, en mayo-junio 2017

Fig. 1. Distribution of phenotypic groups of heterotrophic bacteria isolated from subsurface-level $(5 \mathrm{~m})$ and deep-sea waters of mesophotic reef sites in Cuba. Sampling period: May-June 2017 
Los resultados de la asimilación de diferentes carbohidratos demostraron que el 85.5\% (53/62) de los cultivos fueron capaces de utilizar al menos uno de los carbohidratos evaluados para el crecimiento celular. En particular, se demostró que más del 50.0\% de los aislados de cada nivel crecieron en presencia de estos compuestos, con excepción de los polisacáridos para los aislados del nivel de fondo de los sitios de arrecifes mesofóticos (Fig. 2).

Por otra parte, se encontró que un alto porcentaje de los aislados fueron capaces de utilizar el petróleo para los procesos de multiplicación celular $(53.2 \%, 33 / 62)$ o al menos uno de los compuestos fenólicos ensayados $(50.0 \%, 31 / 62)$. Debe destacarse que 38 de las cepas evaluadas fueron capaces de emplear hidrocarburos y fenoles como fuente de carbono y energía.
Los aislados del grupo de bacilos $\mathrm{G}+$ esporulados de ambos niveles fueron los más representados.

En general, un alto porcentaje de los aislamientos fueron capaces de emplear indistintamente monosacáridos (79.0\%), disacáridos (77.4\%), polisacáridos $(62.9 \%)$, petróleo $(53.2 \%)$ $\mathrm{y}$ fenoles $(50.0 \%)$ como única fuente de carbono y energía. Asimismo, se evidenció que el porcentaje de cepas degradadoras varió en función de la complejidad y accesibilidad del compuesto, independiente de los niveles de la columna de agua, aunque para el nivel subsuperficial el porcentaje de cultivos degradadores fue mayor que para el nivel de fondo (Fig. 2).

Por otra parte, el $96.8 \%(60 / 62)$ de los aislados produjeron enzimas hidrolíticas exocelulares para al menos uno de los sustratos evaluados; aunque

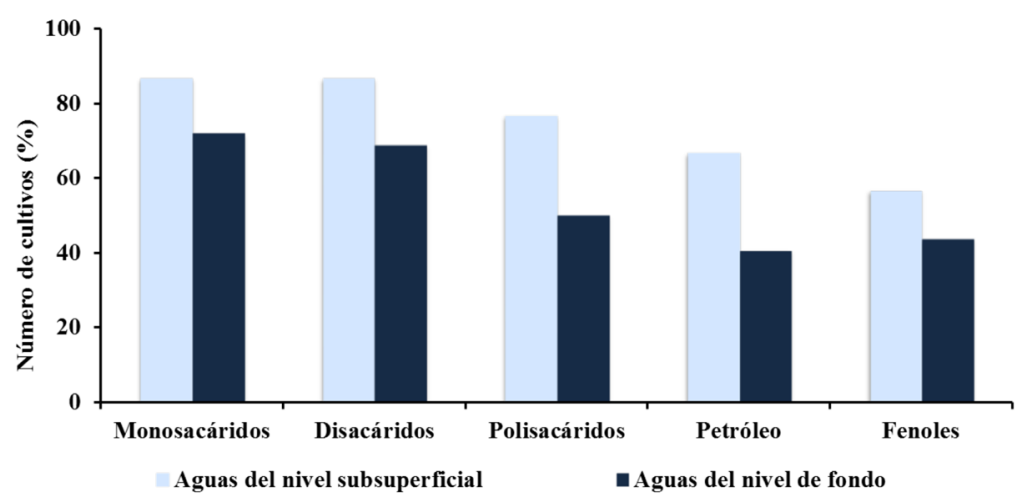

Fig. 2. Porcentaje de aislados de bacterias heterótrofas que asimilan diferentes fuentes de carbono por niveles de la columna de agua

Fig. 2. Percentage of heterotrophic bacteria isolates assimilating different carbon sources as a function of water column level 
resultó notable la baja incidencia de cultivos que hidrolizan la gelatina con respecto al resto de los sustratos (Fig. 3). Los bacilos $\mathrm{G}+$ esporulados y los bacilos G- fueron los que presentaron mayor actividad hidrolítica en ambos niveles, mientras para las aguas del nivel de fondo se detectó un mayor porcentaje de cultivos del grupo de los G- con actividad lipolítica (Fig. 3).

Resulta interesante que el $73.3 \%$ (44/60) de los cultivos con actividad hidrolítica presentaron la capacidad de hidrolizar entre dos o tres de los sustratos evaluados, con una distribución similar entre los aislados de las aguas subsuperficiales $(23 / 29)$ y los de aguas del nivel de fondo (21/31).

A partir de la determinación semicuantitativa de la producción de las enzimas caseinasa, amilasa y lipasa se estableció la clasificación de los cultivos positivos según Ong et al. (2011). En particular, se demostró que 46 cultivos presentaron alta actividad enzimática para al menos una de las enzimas hidrolíticas evaluadas, con la formación de halos de hidrolisis $>8 \mathrm{~mm}$. Asimismo, se detectó que predominó una alta $(>8 \mathrm{~mm})$ y moderada $(>2 ; \leq 8 \mathrm{~mm})$ actividad enzimática en las aguas de ambos niveles, independientemente de la complejidad de los sustratos (Fig. 4). Es de destacar los elevados porcentajes de cultivos del nivel de fondo con alta actividad caseinasa y lipasa $(>8 \mathrm{~mm})$ con respecto a los del nivel subsuperficial (Fig. 4).
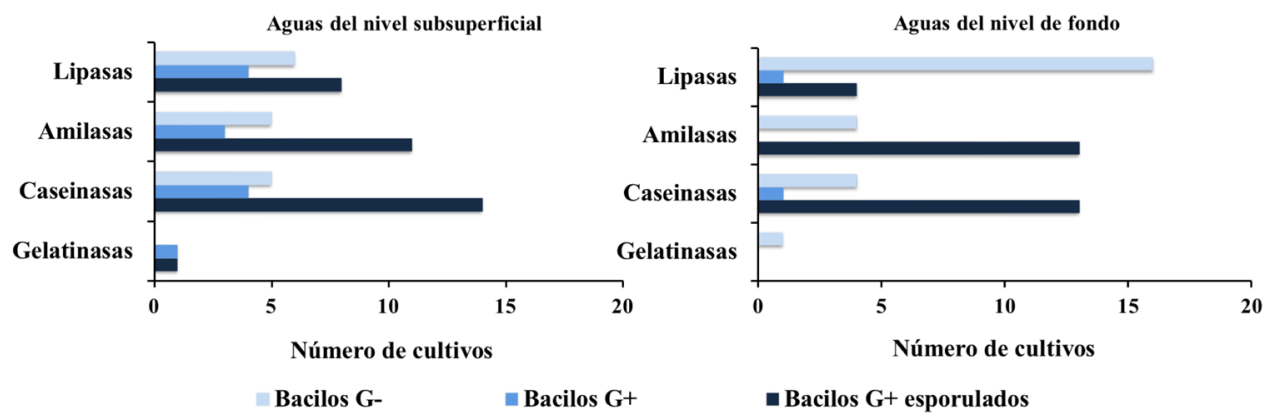

Fig. 3. Distribución de la actividad de enzimas hidrolíticas de acuerdo a los grupos fenotípicos de las bacterias heterótrofas aisladas del nivel subsuperficial y de las aguas profundas de sitios de arrecifes mesofóticos de Cuba

Fig. 3. Activity distribution of hydrolytic enzymes according to phenotypic group of heterotrophic bacteria isolated from subsurface-level and deep-sea waters of Cuban mesophotic reef sites 


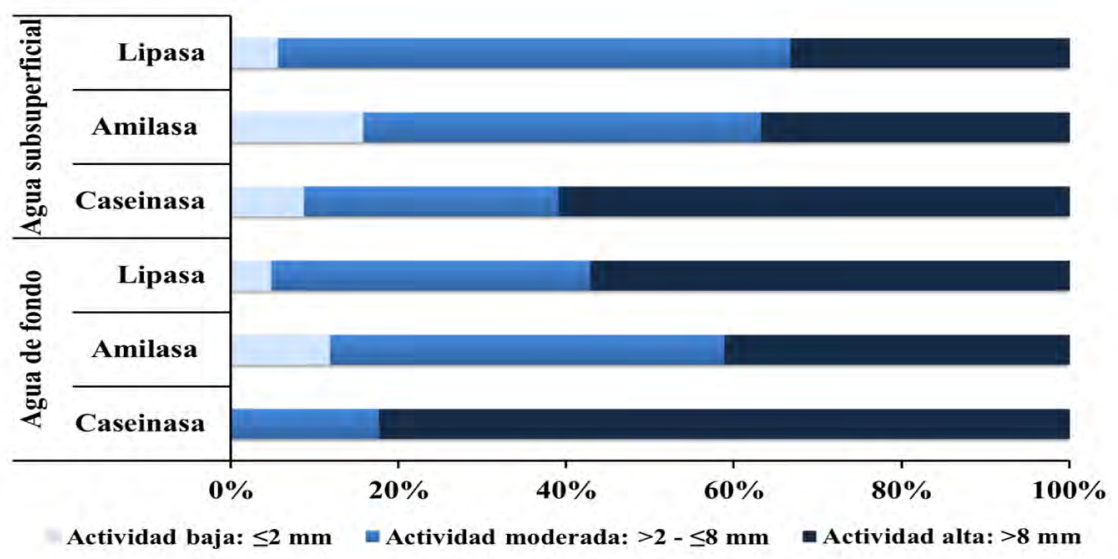

Fig. 4. Distribución del porcentaje de acuerdo a la clasificación semicuantitativa de la producción enzimática de los aislados de agua subsuperficial y aledañas a los arrecifes mesofóticos

Fig. 4. Percentage distribution according to the semi-quantitative classification of the enzymatic production in isolates obtained from subsurface-level and deep-sea waters of Cuban mesophotic reef sites

Del total de aislados un alto porcentaje produjeron sustancias con actividad superficial $(53.2 \%, 33 / 62)$ (Fig. $5 \mathrm{~A}$ ). Los resultados demostraron una distribución similar en el porcentaje de cultivos productores tensioactivos procedentes tanto de las aguas del nivel de fondo $(29.0 \%)$ como del nivel subsuperficial (24.2\%); con valores $\leq 35.0 \mathrm{mN} / \mathrm{m}$ con respecto al control (50.3 mN/m) (Fig. 5A). Debe destacarse que el $27.3 \%$ (9/33) producen compuestos que disminuyen la tensión superficial a valores $\leq 30 \mathrm{mN} / \mathrm{m}$. Asimismo, se encontró que el 60.6\% de los cultivos (20/33) que produjeron compuestos tensioactivos presentan actividad lipolítica; mientras el 24.2\%
(8/33) presentaron además, la capacidad para degradar los hidrocarburos del petróleo.

Por su parte, la evaluación cualitativa de la actividad asparaginasa demostró que el $68.0 \%$ de los cultivos no presentó la actividad (Fig. 5B). Para ambos niveles de la columna de agua se encontraron porcentajes similares de cultivos capaces producir la enzima L-asparaginasa (16.0\%) (Fig. 5B). Debe destacarse que los grupos fenotípicos con mayor incidencia corresponden a los bacilos $\mathrm{G}+$ esporulados $\mathrm{y}$ los bacilos G- para un $35.0 \%(7 / 20)$ y $45.0 \%(9 / 20)$, respectivamente. 
A

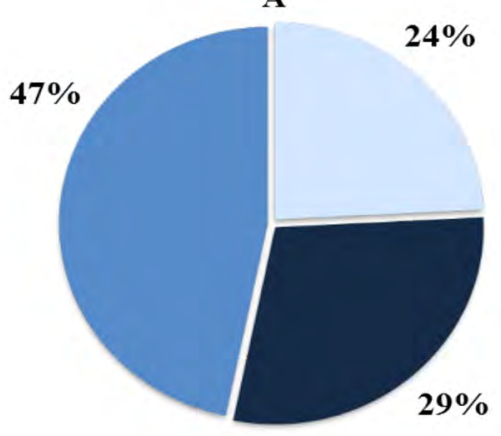

B

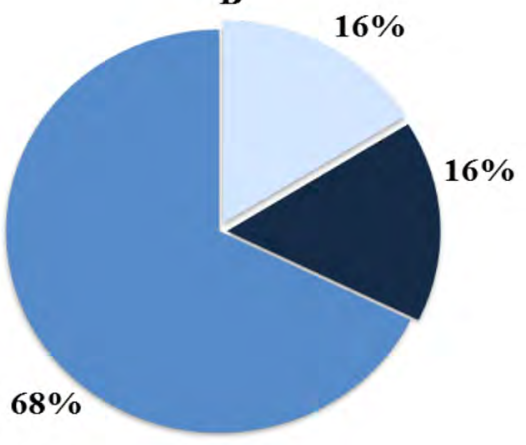

Actividad nivel subsuperficial

Fig. 5. Porcentaje de aislados con capacidades metabólicas de interés biotecnológico aislados de zonas de arrecifes mesofóticos de Cuba. A: Actividad tensioactiva (TS $\leq 35 \mathrm{mN} / \mathrm{m}$ ), B: Actividad L-asparaginasa

Fig. 5. Percentage of isolates exhibiting metabolic capacities of biotechnological interest obtained from Cuban mesophotic reef sites. A: Surfactant activity (surface tension $\leq 35 \mathrm{mN} / \mathrm{m}$ ), B: L-Asparaginase activity

Para la caracterización taxonómica de los aislados se seleccionaron 20 del nivel subsuperficial y 20 de las aguas del nivel de fondo en los sitios de arrecifes mesofóticos, atendiendo a la elevada actividad metabólica, al presentar entre tres y siete de las bioactividades consideradas en este estudio.

Los cultivos seleccionados del grupo de los bacilos Gram- correspondieron al phylum Proteobacteria e identificados como Ochrobactrum anthropi, Aeromonas hydrophila y Photobacterium damselae de las familias Brucellaceae, Aeromonadaceae y Vibronaceae, respectivamente (Fig. 6). Por su parte, todos los cultivos Gram+ esporulados seleccionados por su actividad metabólica correspondieron al phylum Firmicutes, de la familia Bacillaceae y al género Bacillus, con 11 especies para las aguas subsuperficiales y ocho para las aguas del nivel de fondo de los sitios de arrecifes mesofóticos de Cuba (Fig. 6).

En la distribución de las especies por niveles de la columna de agua, $B a-$ cillus acidocaldarius, Bacillus firmus, Bacillus macerans, Bacillus pumilus, Bacillus thuringiensis y Ochrobactrum anthropi fueron aisladas del nivel subsuperficial y no estuvieron presentes en las aguas del nivel de fondo; mientras las especies Bacillus lentus y Bacillus cereus solo se encontraron en las aguas profundas. El resto de las especies identificadas están presentes en ambos niveles (Fig. 6). 


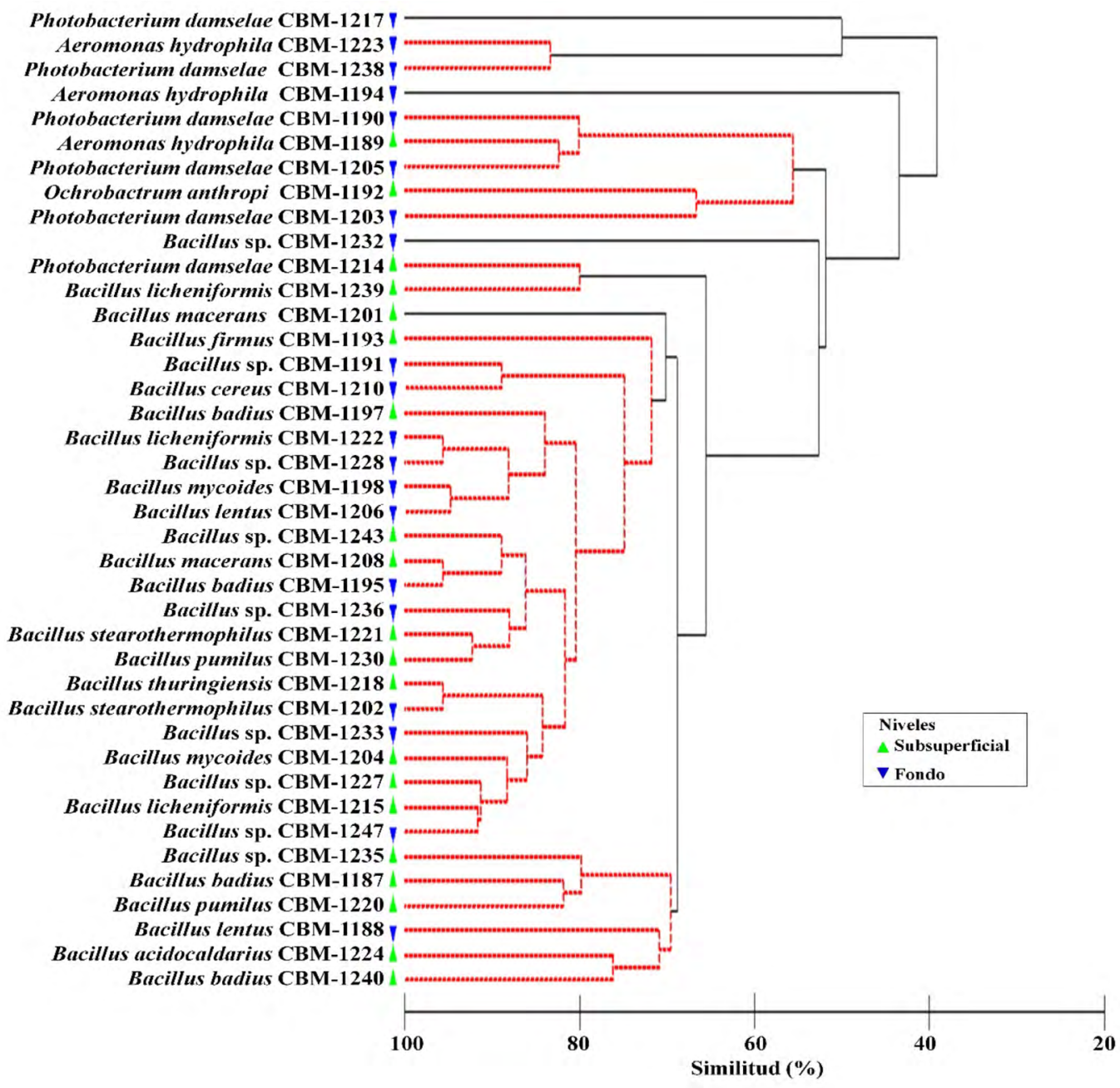

Fig. 6. Agrupamiento de las cepas de bacterias seleccionadas de ambos niveles de la columna de agua a partir de las características fisiológicas y potencialidades biotecnológicas (Índice de similitud de Brays-Curtis)

Fig. 6. Grouping of bacterial strains selected from both water column depth levels based on physiological characteristics and biotechnological potential (Brays-Curtis similarity index)

En el análisis de similitud de las cepas seleccionadas por sus capacidades metabólicas se demostró que la mayoría de los cultivos pertenecientes al género Bacillus se agrupan con un porcentaje de similitud $\geq 70 \%$, independientemente del nivel de la columna de agua de donde fueron aislados. A 
su vez todos los cultivos pertenecientes al phylum Proteobacteria de tres géneros diferentes mostraron menores porcentajes de similitud (Fig. 6).

\section{DISCUSIÓN}

La caracterización macroscópica de los aislados de ambos niveles de la columna de agua en sitios de arrecifes mesofóticos de Cuba, demostró que existe una elevada variedad cultural de bacterias heterótrofas. En particular, las bacterias Gram- mostraron dominancia en las aguas de mayor profundidad; que pudieran incluirse en géneros de las clases Alphaproteobacteria, Gammaproteobacteria, Actinobacteria, Flavobacteria, entre otras; identificados frecuentemente en muestras de agua de niveles pelágicos (Kai et al. 2017; Liu et al. 2018; Cui et al. 2019; Sanz-Sáez et al. 2020). Para las aguas profundas del presente estudio, que abarcan entre los $135-450 \mathrm{~m}$, se ha descrito un predomino de bacterias heterótrofas cultivables pertenecientes al phylum Proteobacteria y que existe un gradiente de diversidad descendente hacia las profundidades, por debajo de la capa fótica (Kai et al. 2017; SanzSáez et al. 2020).

Tanto en la capa fótica como en las de mayor profundidad se ha demostrado que las bacterias heterótrofas están adaptadas a diferentes temperaturas, luz y presión; lo que sugiere que tienen metabolismos versátiles para responder a la disponibilidad de nutrientes. Estas características permiten una amplia distribución vertical y horizontal en los ecosistemas marinos (Jones \& Lennon, 2010).

La asimilación de diferentes carbohidratos como fuentes de carbono demostró la versatilidad metabólica de las bacterias heterótrofas aisladas de ambos niveles de la columna de agua, relacionado con diversas rutas del metabolismo microbiano para la degradación preferencial de estos compuestos (Shon et al. 2006). En general, estas capacidades metabólicas deben estar sustentadas por la presencia de compuestos glucosídicos en los ecosistemas marinos, incluso provenientes del metabolismo microbiano (Sivaperumal et al. 2017; Casillo et al. 2018). Es reconocida la biosíntesis de exopolisacáridos con funciones que incluyen la adhesión y colonización de superficies, la protección de las células bacterianas, entre otras (Dang \& Lovell, 2016; Casillo et al. 2018).

En el presente estudio se demostró la capacidad de degradación bacteriana de compuestos orgánicos tóxicos, como los hidrocarburos del petróleo y fenoles, que evidencian la participación de la comunidad bacteriana en esos procesos y su contribución a la autodepuración de los ecosistemas marinos. Especial interés representan los cultivos que fueron capaces de utilizar ambas sustancias como fuente 
carbono y energía, relacionado con la capacidad de los microorganismos de aprovechar rutas metabólicas comunes para degradar compuestos orgánicos diferentes (Seeger \& Pieper; 2010; Fuentes et al. 2014).

Las bacterias heterótrofas evaluadas también fueron capaces de producir diferentes enzimas hidrolíticas, independientemente de los niveles de la columna de agua, similar a lo informado para diferentes ecosistemas marinos (Liu et al. 2018; Gawas et al. 2019). Resulta interesante la marcada incidencia de cultivos de las aguas profundas con alta actividad enzimática, probablemente relacionada con la naturaleza de compuestos de alto peso molecular y polímeros como proteínas, almidón, ácidos nucleicos, lípidos complejos, entre otros (Arnosti, 2014; Dang \& Lovell, 2016).

Las diferencias en la actividad enzimática entre las bacterias cultivables de cada nivel de la columna de agua evaluada, con una mayor actividad hidrolítica $(>8 \mathrm{~mm})$ para proteasas y lipasas en las aguas profundas con respecto al nivel subsuperficial, debe estar relacionado con la complejidad de los compuestos orgánicos presentes en el agua mar. Liu et al. (2018) informan diferencias sustanciales en las capacidades enzimáticas de las bacterias cultivables a diferentes profundidades, sugiriendo que los carbohidratos probablemente no constituyen un componente mayoritario de la materia orgánica disuelta en las aguas profundas. Así por ejemplo de los aislamientos del nivel de fondo, los bacilos G- predominan por su actividad lipolítica, característico de phylum Proteobacteria de aguas profundas (Liu et al. 2018; Delabary et al. 2020).

Entre los grupos fenotípicos más representados para ambos niveles de los sitios mesofóticos se destacan los bacilos $\mathrm{G}+$ esporulados; donde se incluye el género Bacillus, reconocido como productores de diferentes exoenzimas (Odisi et al. 2012; Lailaja \& Chandrasekaran, 2013; Gawas et al. 2019; Jin et al. 2019).

El elevado porcentaje de aislados bacterianos de las aguas de los sitios mesofóticos de Cuba, capaces de degradar carbohidratos de diferente complejidad y compuestos tóxicos; así como de producir enzimas hidrolíticas, evidencian el papel de las bacterias heterótrofas en la mineralización de la materia orgánica en la columna de agua. En particular, la producción de enzimas hidrolíticas es considerada una adaptación para el aprovechamiento de sustratos disponibles en la columna de agua como fuente de carbono, nutrientes y energía (Arnosti, 2014; Liu et al. 2018), que tiene implicaciones ecológicas relevantes en el reciclaje de materia orgánica y nutrientes, particularmente en el ciclo del carbono en el océano (Becker et al. 2017; Liu et al. 2018). 
Las capacidades metabólicas de los microorganismos para desarrollar procesos de biorremediación también está relacionada con la excreción de enzimas que hidrolizan macromoléculas en monómeros fácilmente degradables (Sivaperumal et al. 2017). Los aislados bacterianos con capacidad de producir enzimas hidrolíticas y degradar hidrocarburos del petróleo y fenoles representan un potencial para el desarrollo de tecnologías eficientes para la biorremediación de compuestos xenobióticos con implicaciones para la salud humana, que incluyen efectos carcinogénicos, mutagénicos y teratogénicos (Ifegwu \& Anyakora, 2015; Chen et al. 2019).

La evaluación de la producción de tensioactivos microbianos estuvo dirigida a la búsqueda de sustancias que disminuyan la tensión superficial del caldo fermentado, que evidencian la síntesis exocelular de estos compuestos y su posible utilización en procesos de biorremediación. En particular, los cultivos que producen agentes con actividad superficial y enzimas específicas como las lipasas, puede ser de gran interés para favorecer tratamientos biológicos en la remoción y biodegradación de hidrocarburos (Karlapudi et al. 2018).

En los últimos años son notables los esfuerzos dirigidos a la bioprospección de compuestos bioactivos a partir de fuentes marinas (Glöckner et al. 2012; Blunt et al. 2018), teniendo en cuenta sus potencialidades como nuevos metabolitos de interés biomédico. La actividad L-asparginasa detectada en los cultivos de los sitios de arrecies mesofóticos guarda relación con lo informado sobre las potencialidades de los microorganismos marinos como productores de esta enzima; las cuales se caracterizan por ser extracelulares, a diferencia de las obtenidas de otros orígenes (Izadpanah Qeshmi et al. 2018). Entre los géneros más representados de fuentes marinas se incluyen Bacillus y Pseudomonas (Rudrapati \& Audipudi, 2015; Izadpanah Qeshmi et al. 2018)

La enzima L-asparaginasa es considerada un producto natural para el tratamiento oncogénico, debido a que las células tumorales dependen de una fuente exógena de L-asparagina, a diferencia de las células normales (Rudrapati \& Audipudi, 2015). En particular, la síntesis microbiana de esta enzima como metabolito secundario ha demostrado actividad antineoplásica y con amplio espectro de actividad antitumoral (Jain et al. 2012).

La caracterización taxonómica de los cultivos de bacterias seleccionados por sus potencialidades demostró conectividad entre las aguas procedentes de las diferentes profundidades, teniendo en cuenta la ubicuidad de los grupos bacterianos identificados. Esta particularidad ha sido demostrada a partir del análisis filogenético de bacterias cultivables en la columna de 
agua a diferentes profundidades oceánicas (Sanz-Sáez et al. 2020).

El predominio del género $\mathrm{Baci-}$ llus entre los aislados de ambos niveles de la columna de agua, debe estar relacionado con la amplia distribución de este grupo en diferentes ecosistemas marinos, ya que este género se caracteriza por presentar esporas como estructura de resistencia y supervivencia ante cambios ambientales; así como una alta versatilidad metabólica para su adaptación a las condiciones extremas (Izadpanah Qeshmi et al. 2018; Gawas et al. 2019). También Lugioyo et al. (2010) refirieron un predominio de la familia Bacillaceae, específicamente al género Bacillus, en muestras de aguas oceánicas adyacentes a Cuba. Para ese estudio se demostró que las capacidades metabólicas de una misma especie de bacterias pueden variar de acuerdo al grado de riqueza de los ecosistemas.

La literatura especializada refiere que las especies de este género son productores de sustancias biológicamente activas, entre las que se encuentran antimicrobianos, tensioactivos, enzimas hidrolíticas y degradadoras de los hidrocarburos del petróleo (Sardessai \& Bhosle, 2002; Odisi et al. 2012).

En general en este estudio se obtuvo un elevado porcentaje de cultivos con diversas capacidades metabólicas, que constituyen un recurso potencial de aplicación industrial. Así, por ejemplo, la obtención de metabolitos microbianos resulta sencilla y sus rendimientos pueden ser incrementados optimizando las condiciones de cultivo o a través de la modificación del genoma microbiano (Glöckner et al. 2012; Liu et al. 2013).

A partir de los resultados de esta investigación se logró actualizar el conocimiento de la diversidad bacteriana marina; y en especial de las aguas aledañas a los arrecifes mesofóticos de Cuba, que no habían sido estudiados con anterioridad. En particular, los aislados bacterianos procedentes de aguas profundas resultaron de interés, teniendo en cuenta las condiciones en que se desarrollan, como alta salinidad, baja disponibilidad de oxígeno, $\mathrm{pH}$ y presiones hidrostáticas extremas, entre otras; de manera que sus metabolitos pudieran presentar novedosas estructuras moleculares con interesantes propiedades de aplicación industrial y biotecnológica (Ramlath et al. 2018; Trincone, 2018; Jin et al. 2019).

\section{AGRADECIMIENTOS}

Esta investigación ha sido posible por el financiamiento del Instituto para la Exploración, Investigación y Tecnología Oceánica de la Universidad Atlántica de la Florida y el Instituto de Estudios Marinos y Atmosféricos de la Universidad de Miami. Además, la investigación fue cofinanciada por el Programa Nacional de Ciencia, 
Tecnología e Innovación "Uso sostenible de los componentes de la diversidad biológica en Cuba", a través del Proyecto Arrecifes mesofóticos de Cuba. También agradecemos el trabajo de los evaluadores y editores de la revista por su contribución a la calidad del presente manuscrito.

\section{BIBLIOGRAFÍA}

Arnosti, C. (2014). Patterns of microbially driven carbon cycling in the ocean: links between extracellular enzymes and microbial communities. $A d$. Oceanogra., 2014, 1-12. https://doi. org/10.1155/2014/706082

Atlas, R. M. (2010). Handbook of microbiological media. EE. UU.: CRC Press.

Becker, S., Scheffel, A., Polz, M. F. \& Hehemann, J. H. (2017). Accurate quantification of laminarin in marine organic matter with enzymes from marine microbes. Appli. Environ. Microbiol., 83(9). https://doi.org/10.1128/ AEM.03389-16

Benson, T. (2001). Microbiological Applications Laboratory Manual in General Microbiology. 8th Edition. EE. UU.: The McGraw-Hill.

Blunt, J. W., Carroll, A. R., Copp, B. R., Davis, R. A., Keyzers, R. A. \& Prinsep, M. R. (2018). Marine natural products. Nat. Prod. Rep., 35(1), 8-53. https:// doi.org/10.1039/c7np00052a

Casillo, A., Lanzetta, R., Parrilli, M. \& Corsaro, M. M. (2018). Exopolysaccharides from marine and marine extremophilic bacteria: structures, properties, ecological roles and applications. Mar. Drugs, 16(2), 69. https://doi.org/10.3390/ md16020069
Chen, Y.-Y., Kao, T.-W., Wang, C.-C., Chen, Y.-J., Wu, C.-J., Lai, C.-H. \& Chen, W.-L. (2019). Exposure to polycyclic aromatic hydrocarbons and risk of disability among an elderly population. Environ. Sci. Pollut. Res., https://doi. org/10.1007/s11356-019-04498-3

Clarke, K. R. \& Warwick, R. M. (2001). Change in Marine Communities: An approach to statistical analysis and interpretation (2nd ed.). United Kingdom: PRIMER-E Ltd.

Cui, Y., Chun, S. J., Baek, S. H., Lee, M., Kim, Y., Lee, H. G., ... \& Oh, H. M. (2019). The water depth-dependent co-occurrence patterns of marine bacteria in shallow and dynamic Southern Coast, Korea. Sci. Rep., 9(1), 1-13. https://doi. org/10.1038/s41598-019-45512-5

Dang, H. \& Lovell, C. R. (2016). Microbial surface colonization and biofilm development in marine environments. Microbiol. Mol. Biol. Rev., 80(1), 91-138. $\quad$ https://doi.org/10.1128/ MMBR.00037-15

Delabary, G. S., Silva, M. C. D., Silva, C. S. D., Baratieri, L. Z., Melo, T. M. D., Stramosk, C. A, ... \& Silva, M. A. C. D. (2020). Influence of temperature and culture media on growth and lipolytic activity of deep-sea Halomonas sulfidaeris LAMA 838 and Marinobacter excellens LAMA 842.0 Ocean Coast. Res., 68(e20282) 1-11. https://dx.doi. org/10.1590/S2675-28242020068282

Dias, A. C., Andreote, F. D., Dini-Andreote, F., Lacava, P. T., Sá, A. L., Melo, I. S., ... \& Araújo, W. L. (2009). Diversity and biotechnological potential of culturable bacteria from Brazilian mangrove sediment. W. J. Microbiol. Biotechnol., 25(7), 1305-1311. https://doi.org/10.1007/ s11274-009-0013-7 
Finnerty, W. R. (1994). Biosurfactants in environmental biotechnology. Current Opinion Biotechnol., 5(3), 291-295. https://doi. org/10.1016/0958-1669(94)90031-0

Fuentes, S., Méndez, V., Aguila, P. \& Seeger, M. (2014). Bioremediation of petroleum hydrocarbons: catabolic genes, microbial communities, and applications. Applied Microbiol. Biotechnol., 98(11), 4781-4794. https://doi. org/10.1007/s00253-014-5684-9

Gawas, V. S., Shivaramu, M. S., Damare, S. R., Pujitha, D., Meena, R. M. \& Shenoy, B. D. (2019). Diversity and extracellular enzyme activities of heterotrophic bacteria from sediments of the Central Indian Ocean Basin. Sci. Rep., 9(1), 1-9. https://doi.org/10.1038/ s41598-019-45792-x

Gilbert, J. A., Steele, J. A., Caporaso, J. G., Steinbrück, L., Reeder, J., Temperton, B., ... \& Somerfield, P. (2012). Defining seasonal marine microbial community dynamics. ISME J., 6(2), 298-308. https://doi.org/10.1038/ismej.2011.107

Glöckner, F. O., Stal, L. J., Sandaa, R.-A., Gasol, J. M., O’Gara, F., Hernandez, F., ... \& Pitta, P. (2012). Marine Microbial Diversity and its role in Ecosystem Functioning and Environmental Change. In J. B. Calewaert \& N. McDonough (Eds.), Marine Board Position Paper 17 (pp. 5-77) Belgium: Marine Board-ESF. https://doi.org/10.13140/ RG.2.1.5138.6400

Harrigan, W. F. \& McCance, M. E. (1968). Métodos de laboratorio en microbiología. España: Academia.

Ifegwu, O. C. \& Anyakora, C. (2015). Polycyclic aromatic hydrocarbons: part I. Exposure. In G. Makowki (Ed.), $A d$ vances in Clinical Chemistry (pp. 277304). EE.UU.: Academic Press. https:// dx.doi.org/10.1016/bs.acc.2015.08.001
Izadpanah Qeshmi, F., Homaei, A., Fernandes, P. \& Javadpour, S. (2018). Marine microbial L-asparaginase: Biochemistry, molecular approaches and applications in tumor therapy and in food industry. Microbiol. Res., 208, 99-112. https:// doi.org/10.1016/j.micres.2018.01.011

Jain, R., Zaidi, K. U., Verma, Y. \& Saxena, P. (2012). L-asparaginase: A promising enzyme for treatment of acute lymphoblastic leukiemia. People's J. Sci. Res., 5(1), 29-35.

Jin, M., Gai, Y., Guo, X., Hou, Y. \& Zeng, R. (2019). Properties and applications of extremozymes from deep-sea extremophilic microorganisms: A mini review. Mar. Drugs, 17(12), 656. https://doi. org/10.3390/md17120656

Jones, S. E. \& Lennon, J. T. (2010). Dormancy contributes to the maintenance of microbial diversity. Proc. Natl. Acad. Sci., 107(13), 5881-5886. https://doi. org/10.1073/pnas.0912765107

Kai, W., Peisheng, Y., Rui, M., Wenwen, J. \& Zongze, S. (2017). Diversity of culturable bacteria in deep-sea water from the South Atlantic Ocean. Bioengineered, 8(5), 572-584. https://doi.org/10.1 080/21655979.2017.1284711

Karlapudi, A. P., Venkateswarulu, T. C., Tammineedi, J., Kanumuri, L., Ravuru, B. K., Dirisala, V. \& Kodali, V. P. (2018). Role of biosurfactants in bioremediation of oil pollution-a review. Pet. 4(3), 241-249. https://doi.org/10.1016/j. petlm.2018.03.007

Lailaja, V. P. \& Chandrasekaran, M. (2013). Detergent compatible alkaline lipase produced by marine Bacillus smithii BTMS 11. World J. Microbiol. Biotechnol., 29(8), 1349-1360. https://doi. org/10.1007/s11274-013-1298-0

Liu, L., Yang, H., Shin, H. D., Chen, R. R., Li, J., Du, G. \& Chen, J. (2013). How to achieve high-level expression of 
microbial enzymes: strategies and perspectives. Bioengineered, 4(4), 212-223. https://doi.org/10.4161/bioe.24761

Liu, Q., Fang, J., Li, J., Zhang, L., Xie, B. B., Chen, X. L. \& Zhang, Y. Z. (2018). Depth-resolved variations of cultivable bacteria and their extracellular enzymes in the water column of the New Britain Trench. Front. Microbiol., 9, 135. https://doi.org/10.3389/ fmicb.2018.00135

Loya, Y., Eyal, G., Treibitz, T., Lesser, M. P. \& Appeldoorn, R. (2016). Theme section on mesophotic coral ecosystems: advances in knowledge and future perspectives. Coral Reefs, 35(1), 1-9. https://doi.org/10.1007/ s00338-016-1410-7

Lugioyo, G. M., Coto, O., Álvarez, C. \& Espinosa, G. (2010). Bacillus species in the oceanic waters adjacent to Cuba: Association between their distribution and metabolic activity. Rev. Cien. Mar. Cost., 2(1), 61-72. https://doi. org/10.15359/revmar.2.5

Lugioyo, G. M., González, D. \& García, I. (2020). Evaluación de la calidad del agua de los arrecifes del golfo de Cazones, sur de Cuba, a partir de algunos indicadores microbiológicos y químicos. Rev. Cien. Mar. Cost., 12(1), 9-26. https://dx.doi.org/10.15359/ revmar.12-1.1

Miravet, M. E. (2003). Abundancia, actividad $y$ diversidad de las bacterias heterótrofas en el Golfo de Batabanó y su uso como indicadoras ambientales. (Tesis de doctorado no publicada). Universidad de La Habana, Cuba.

Miravet, M. E., Lugioyo, M., Loza, S., Enríquez, D., Delgado, Y., Carmenate, M. \& Pérez, D. (2009). Procedimientos para el Monitoreo de la Calidad Ambiental en la Zona Marino Costera a partir de Microorganismos. República Dominicana: Ediciones Centenario.

Miravet, M. E., Martínez-Daranas, B. \& Penie, I. (1994). Indicadores microbiológicos del estado de salud de los arrecifes que bordean el archipiélago Sabana-Camagüey. Cuba: Instituto de Oceanología.

Odisi, E. J., Silvestrin, M. B., Takahashi, R. Y. U., da Silva, M. A. C. \& Lima, A. O. D. S. (2012). Bioprospection of cellulolytic and lipolytic South Atlantic deep-sea bacteria. Electron. J. Biotechnol., 15(5). https://doi.org/10.2225/ vol15-issue 5-fulltext-17

Olson, J. B. \& Kellogg, C. A. (2010). Microbial ecology of corals, sponges, and algae in mesophotic coral environments. FEMS Microbiol. Ecol., 73(1), 17-30. https://doi. org/10.1111/j.1574-6941.2010.00862.x

Ong, K. S., Chin, H. S. \& Teo, K. C. (2011). Screening of antibiotic sensitivity, antibacterial and enzymatic activities of microbes isolated from ex-tin mining lake. African J. Microbiol. Res., 5(17), 2460-2466. https://doi.org/10.5897/ AJMR11.170

Oppenheimer, C. H. \& ZoBell, C. E. (1952). The growth and viability of sixty-three species of marine bacteria as influenced by hydrostatic pressure. J. Mar. Res., 11(1), 10-18.

Prabhu, R. H., Bhise, K. S. \& Patravale, V. B. (2017). Marine enzymes in cancer: a new paradigm. In S. Kim \& F. Toldrá (Eds.), Advances in Food and Nutrition Research. Marine Enzymes Biotechnology: Production and Industrial Applications, Part III - Application of Marine Enzymes (pp. 1-14). EE. UU.: Academic Press.

Ramlath, L., Keerthana, P. P., Safvana Fathima, P. \& Mashhoor, K. (2018). Bacteria from Coral Ecosystem of Kiltan 
Island, Lakshadweep: Resource for Hydrolytic Enzymes. Int. J. Cell Sci. Biotechnol., 7, 1-9.

Roman, J. (2018). The ecology and conservation of Cuba's coastal and marine ecosystems. Bull. Mar. Sci., 94(2), 149-169. https://doi.org/10.5343/ bms.2017.1164

Rudrapati, P. \& Audipudi, A. V. (2015). Characterization and bioprocessing of oncolytic enzyme-L-asparaginase isolated from marine bacillus AVP 14. Int. J. Pharm. Sci. Rev. Res., 30(2), 195-201.

Sanz-Sáez, I., Salazar, G., Sánchez, P., Lara, E., Royo-Llonch, M., Sà, E. L., .... \& Acinas, S. G. (2020). Diversity and distribution of marine heterotrophic bacteria from a large culture collection. BMC Microbiol., 20(1), 1-16. https:// doi.org/10.1186/s12866-020-01884-7

Sardessai, Y. N. \& Bhosle, S. (2002). Organic solvent-tolerant bacteria in mangrove ecosystem. Current Sci., 82(6), 622-623.

Seeger, M. \& Pieper, D. H. (2010). Genetics of biphenyl biodegradation and co-metabolism of PCBs. In K. N. Timmis (Ed.), Handbook of hydrocarbon and Lipid Microbiology (pp. 1180-1198). Germany: Springer-Verlag. https://doi. org/10.1007/978-3-540-77587-4_82

Shon, H. K., Vigneswaran, S. \& Snyder, S. A. (2006). Effluent organic matter (EfOM) in wastewater: constituents, effects, and treatment. Crit. Rev. Environ. Sci. Technol., 36(4), 327-374. https://doi. org/10.1080/10643380600580011
Sivaperumal, P., Kamala, K., \& Rajaram, R. (2017). Bioremediation of industrial waste through enzyme producing marine microorganisms. In $\mathrm{S}$. Kim \& F. Toldrá (Eds.), Advances in Food and Nutrition Research. Marine Enzymes Biotechnology: Production and Industrial Applications, Part III - Application of Marine Enzymes (pp. 165-179). EE. UU.: Academic Press.

Sivasankar, P., Sugesh, S., Vijayan, P., Sivakumar, K., Vijayalakshmi, S., Balasubramanian, T. \& Mayavu, P. (2013). Efficient production of L-asparaginase by marine Streptomyces sp. isolated from Bay of Bengal, India. African J. Microbiol. Res., 7(31), 4015-4021.

Trincone, A. (2018). Update on marine carbohydrate hydrolyzing enzymes: biotechnological applications. Molecules, 23(4), 901. https://doi.org/10.3390/ molecules 23040901

Vela, G. R. \& Ralston, J. R. (1978). The effect of temperature on phenol degradation in wastewater. Canadian J. Microbiol., 24(11), 1366-1370. https://doi. org/10.1139/m78-219

Ward, C. S., Yung, C. M., Davis, K. M., Blinebry, S. K., Williams, T. C., Johnson, Z. I. \& Hunt, D. E. (2017). Annual community patterns are driven by seasonal switching between closely related marine bacteria. ISME J., 11(6), 1412-1422. https://doi.org/10.1038/ ismej. 2017.4 